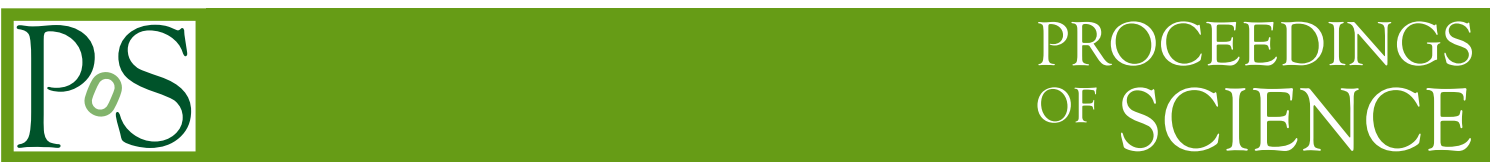

\title{
Strange quark contribution to the nucleon form factor
}

\section{S. Baunack}

Institut für Kernphysik, Universität Mainz

baunack@kph.uni-mainz.de

According to QCD, the nucleon is made up of valence quarks, sea quarks and gluons. Concerning the quark sea, also strange quarks can contribute to the nucleon properties. Parity violating electron scattering offers a tool to investigate the strange quark contribution to the nucleon form factors parameterized by the strange form factors $G_{E}^{s}$ and $G_{M}^{s}$. The theoretical framework to access these strange form factors is outlined here and an overview of the existing world data is given. The measurements performed by the A4 collaboration at the electron accelerator facility MAMI are described here in more details and preliminary results are reported.

8th Conference Quark Confinement and the Hadron Spectrum September 1-6 2008

Mainz, Germany

\footnotetext{
* Speaker.
} 
In the framework of QCD, valence quarks, sea quarks and gluons contribute to the properties of the nucleon. Strange quark contributions are of a specific interest since this is a pure sea quark effect. Various matrix elements may be used for an investigation of strange quark effects [1]. The scalar matrix element $<p|\bar{s} s| p>$ gives information about the strange quark contribution to the mass of the nucleon[2], the axial element $<p\left|\bar{s} \gamma_{\mu} \gamma_{5} s\right| p>$ about the strange quark contribution to the spin of the nucleon[3], while the vector element $\left\langle p\left|\bar{s} \gamma_{\mu} s\right| p>\right.$ provides access to the contribution of strange quarks to the vector current of the nucleon and hence to vector form factors of the nucleon. $[5,4]$ To separate the contribution of the individual quark flavors to the electromagnetic form factors, one can make a flavor decomposition omitting the heavier quarks $c, b$ and $t$,

$$
G_{E, M}^{p, n}=\sum_{f=u, d, s} q_{f} G_{E, M}^{p / n, f}=\frac{2}{3} G_{E, M}^{p / n, u}-\frac{1}{3} G_{E, M}^{p / n, d}-\frac{1}{3} G_{E, M}^{p / n, s}
$$

where $G_{E, M}^{p / n, f}$ denotes the contribution of the quark flavor $f$ to the electric or magnetic form factor of the proton or the neutron. The factors $2 / 3$ and $-1 / 3$ reflect the electric charges of the corresponding quarks. Using isospin symmetry, one gets $G_{E, M}^{p, u}=G_{E, M}^{n, d} \equiv G_{E, M}^{u}, G_{E, M}^{p, d}=G_{E, M}^{n, u} \equiv G_{E, M}^{d}$ and $G_{E, M}^{p, u}=G_{E, M}^{n, d} \equiv G_{E, M}^{u}$. The weak interaction provides additional information. When scattering longitudinally polarized electrons off unpolarized protons, the interference between tree level electromagnetic and weak amplitudes leads to a parity violating asymmetry in the elastic scattering cross section of right- and left-handed electrons $\sigma^{+}, \sigma^{-}: A_{P V}=\left(\sigma^{+}-\sigma^{-}\right) /\left(\sigma^{+}+\sigma^{-}\right)$. This asymmetry can be expressed by the known electromagnetic form factors of proton and neutron, by the weak axial form factor of the proton $\tilde{G}_{A}^{p}$ and the unknown strange form factors $G_{E}^{s}$ and $G_{M}^{s}$ as a sum of three terms, $A_{P V}=A_{V}+A_{S}+A_{A}: A_{V}$ represents the vector coupling on the proton vertex where the strangeness contribution has been taken out, $A_{S}$ contains the strange quark vector contribution, and $A_{A}$ represents the axial coupling on the proton vertex[5]:

$$
\begin{array}{r}
A_{P V}=-a \rho_{e q}^{\prime}\left(\left(1-4 \hat{\kappa}_{e q}^{\prime} \hat{s}_{Z}^{2}\right)-\frac{\varepsilon G_{E}^{p} G_{E}^{n}+\tau G_{M}^{p} G_{M}^{n}}{\varepsilon\left(G_{E}^{p}\right)^{2}+\tau\left(G_{M}^{p}\right)^{2}}\right) \\
A_{S}=a \rho_{e q}^{\prime} \frac{\varepsilon G_{E}^{p} G_{E}^{s}+\tau G_{M}^{p} G_{M}^{s}}{\varepsilon\left(G_{E}^{p}\right)^{2}+\tau\left(G_{M}^{p}\right)^{2}} \\
A_{A}=a \frac{\left(1-4 \hat{s}_{Z}^{2}\right) \sqrt{1-\varepsilon^{2}} \sqrt{\tau(1+\tau)} G_{M}^{p} \tilde{G}_{A}^{p}}{\varepsilon\left(G_{E}^{p}\right)^{2}+\tau\left(G_{M}^{p}\right)^{2}}
\end{array}
$$

with $a=\frac{G_{\mu} Q^{2}}{4 \pi \alpha \sqrt{2}}, G_{\mu}$ the Fermi coupling constant, $\alpha$ the fine structure constant, $\tau=\frac{Q^{2}}{4 M_{p}^{2}}, Q^{2}$ the negative squared momentum transfer and $M_{p}$ the proton mass, $\varepsilon=\left[1+2(1+\tau) \tan ^{2} \frac{\Theta}{2}\right]^{-1}, \Theta$ the scattering angle in the laboratory frame, $\hat{s}_{Z}^{2}\left(M_{Z}\right)=0.23119(14)[6] . \rho_{e q}^{\prime}$ and $\hat{\kappa}_{e q}^{\prime}$ include the electroweak radiative corrections evaluated in the $\overline{M S}$ scheme. Hence measuring the parity violating asymmetry $A_{P V}$ yields one linear combination of strange electric and magnetic form factor $G_{E}^{s}+$ $\alpha G_{M}^{s}$ where $\alpha$ depends on the kinematics. There are various theoretical approaches to the strange quark contribution to the vector current of the proton, including quark model estimates[11], quark soliton models[12, 13] or quenched lattice calculations[14]. 


\section{Existing world data}

A measurement of $A_{P V}$ at forward angles with a proton target yields a linear combination of $G_{E}^{s}$ and $G_{M}^{s}$, a measurement at forward angles with a helium target gives a measurement of $G_{E}^{s}$ alone, at backward angles measurements on a proton and a deuterium target give two linear combinations of $G_{M}^{s}$ and $\tilde{G}_{A}^{p}$. Table 1 gives an overview over the experiments performed so far. Currently the most information is available at the negative squared momentum transfer

\begin{tabular}{c|l|l|l|l} 
& $\begin{array}{l}\mathrm{p} \text { target } \\
\text { forward angle }\end{array}$ & $\begin{array}{l}\mathrm{p} \text { target } \\
\text { backward angle }\end{array}$ & $\begin{array}{l}{ }^{4} \mathrm{He} \text { target } \\
\text { forward angle }\end{array}$ & $\begin{array}{l}\mathrm{d} \text { target } \\
\text { backward angle }\end{array}$ \\
\hline SAMPLE & & $0.1 \mathrm{GeV}^{2}$ & & $\begin{array}{l}0.04 \mathrm{GeV}^{2} \\
0.1 \mathrm{GeV}^{2}\end{array}$ \\
\hline Happex & $\begin{array}{l}0.1 \mathrm{GeV}^{2} \\
0.48 \mathrm{GeV}^{2} \\
0.6 \mathrm{GeV}^{2}\end{array}$ & & $0.1 \mathrm{GeV}^{2}$ & \\
\hline $\mathrm{A} 4$ & $\begin{array}{l}0.1 \mathrm{GeV}^{2} \\
0.23 \mathrm{GeV}^{2} \\
0.6 \mathrm{GeV}^{2}\end{array}$ & $0.23 \mathrm{GeV}^{2}$ & & $0.23 \mathrm{GeV}^{2}$ \\
\hline$G^{0}$ & $\begin{array}{l}(0.12 \ldots 1) \mathrm{GeV}^{2} \\
0.23 \mathrm{GeV}^{2}\end{array}$ & & $0.23 \mathrm{GeV}^{2}$ \\
$0.62 \mathrm{GeV}^{2}$ & & $0.62 \mathrm{GeV}^{2}$ \\
\hline
\end{tabular}

Table 1: PV experiments and their kinematical conditions and targets. A wide range of momentum transfers and scattering angles is already covered. The data points shown in italic are scheduled for the measurement in 2009.

$Q^{2}=0.1 \mathrm{GeV}^{2}$. The SAMPLE collaboration [7], the A4 collaboration [15], the $G^{0}$ collaboration [9] and the HAPPEX collaboration [10] have published results. The disentangling of the strange form factors lead to the values of $G_{E}^{s}=-0.005 \pm 0.019$ and $G_{M}^{s}=0.18 \pm 0.27[10]$.

At $Q^{2}=0.23 \mathrm{GeV}^{2}$ there are results for forward angle measurements published by the collaborations $\mathrm{A} 4$ [8] and $G^{0}$ [9]. Backward angle measurements were already performed by these two collaborations and their results are expected soon to be published. A preliminary result for the A4 collaboration will be presented in the next chapters allowing a disentangling of the strange electric and magnetic form factors for this momentum transfer. The Happex and A4 experimental programs at $Q^{2} \approx 0.6 \mathrm{GeV}^{2}$ will take place in 2009 .

\section{The A4 experimental setup}

The A4 experiment[16] takes place at the electron accelerator facility MAMI at Mainz. A superlattice photocathode delivers a polarized electron beam with an intensity of $20 \mu \mathrm{A}$ and an average polarization of about $80 \%$. The electron beam is accelerated up to $E=1.5 \mathrm{GeV}$. Two polarimeters are in the beamline of the A4 experiment, a Compton Backscatter polarimeter [17] and a Transmission Compton polarimeter [18]. For the measurements presented here weekly polarization measurements were performed by the Moeller polarimeter of the A1 collaboration and frequently measurements by a Mott polarimeter situated close to the electron source resulting in a $4 \%$ uncertainty of the beam polarization. The helicity of the electrons are changed every $20 \mathrm{~ms}$ 
following a randomly selected pattern of either "+-+" or "--+-". To suppress helicity correlated false asymmetries which may arise due to different beam position, angle, intensity or energy for the two helicity states, several feedback stabilization systems are installed along the way of the electrons from the accelerator to the target. The liquid hydrogen target [19] is $23.3 \mathrm{~cm}$ long in case of the backward angle measurement resulting in a luminosity of $L \approx 1.2 \cdot 10^{38} \mathrm{~cm}^{-2} \mathrm{~s}^{-1}$.

The scattered electrons are detected in a homogenous electromagnetic calorimeter that consists of 1022 lead fluoride crystals. Lead fluoride is a pure Cherenkov radiator and hence intrinsically fast. The whole calorimeter can cope with event rates of $100 \mathrm{MHz}$, the energy resolution is about $\Delta E / E=3.9 \% / \sqrt{E[\mathrm{GeV}]}$. The covered angles are $2 \pi$ in the azimuthal range and $30^{\circ} \leq \theta \leq 40^{\circ}$ (forward configuration) or $140^{\circ} \leq \theta \leq 150^{\circ}$ (backward configuration) in the polar range, resulting in a covered solid angle of $\Delta \Omega=0.62 \mathrm{sr}$. In the backward angle configuration there are 72 plastic scintillators installed in front of the $\mathrm{PbF}_{2}$ crystals. They enable a separation of neutral from charged particles, i.e. in our case photons from $\pi^{0}$-decay from scattered electrons which cannot be distinguished by the $\mathrm{PbF}_{2}$ calorimeter itself because the photons produce in the crystals electromagnetic showers very similar to those coming from electrons.

\section{Result of the A4 backward angle measurement}

To match the momentum transfer of $Q^{2}=0.23 \mathrm{GeV}^{2}$, a beam energy of $E=315.1 \mathrm{MeV}$ was chosen. About 1100 hours. of asymmetry data was taken, half of it with a half-wave plate inserted at the electron source reversing the electron helicity, and approximately $3 \cdot 10^{12}$ elastic events were used to determine the parity violating asymmetry. Fig. 1 shows on the left the two asymmetries

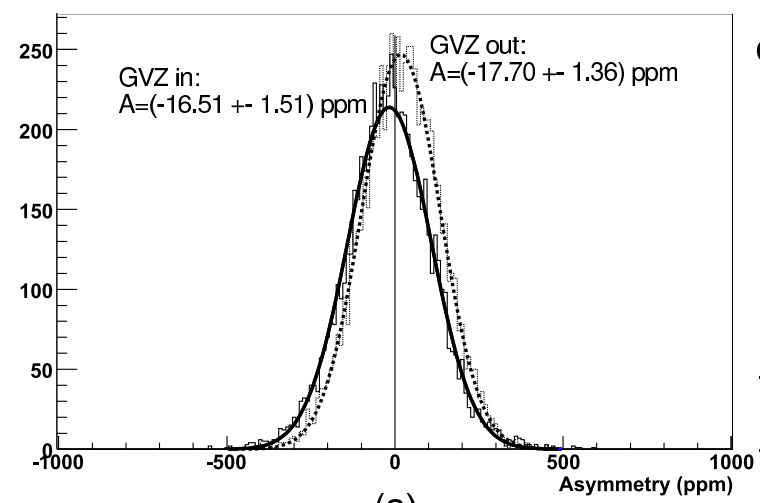

(a)

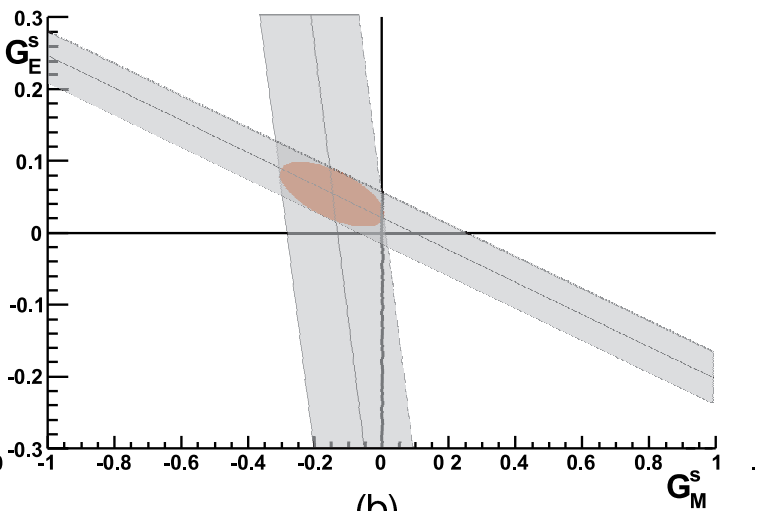

(b)

Figure 1: (a) Asymmetries of background corrected asymmetries for more than 120005 min. runs with respect to the state of the half-wave plate (OUT or IN). One can observe well the sign flip, the asymmetries for OUT and IN agree within the $1 \sigma$-range. (b) 1- $\sigma$ Error bands for the linear combination of $G_{E}^{s}$ and $G_{M}^{s}$ for $Q^{2}=0.23 \mathrm{GeV}^{2}$ for the A4 backward angle measurement (preliminary, the one with the steeper slope) and the A4 forward angle measurement. The overlap region indicates the $68 \%$ C.L. constraints in the $G_{E}^{s}-G_{M}^{s}$ plane.

where the halfwave-plate was out or in respectively. The asymmetries follow a Gaussian distribution. One can clearly observe a sign flip, the two asymmetries agree in size within the uncertainty. 
The combined asymmetry with the sign flip taken into account is $A_{P V}=(-17.41 \pm 1.21) \cdot 10^{-6}$ (preliminary). The asymmetry without strangeness expectation is $A_{0}=(-16.27 \pm 1.22) \cdot 10^{-6}$ where the largest contribution to the error arises from the uncertainty in the weak axial form $\tilde{G}_{A}^{p}$. From these two values we extract a linear combination of the strange form factors: $G_{M}^{s}+0.27 G_{E}^{s}=$ $-0.126 \pm 0.158$. Together with the A4 forward angle measurement, one can disentangle the form factors for $Q^{2}=0.23 \mathrm{GeV}^{2}: G_{E}^{s}=0.055 \pm 0.046$ and $G_{M}^{s}=-0.148 \pm 0.164$ (preliminary).

\section{Conclusions and outlook}

Over the last decade, parity violating electron scattering has become a reliable tool to determine the contribution of strange sea quarks to the vector current of the nucleon. The measurements at $Q^{2}=0.1 \mathrm{GeV}^{2}$ and at $Q^{2}=0.23 \mathrm{GeV}^{2}$ put rather tight constraints to these contributions at these low momentum transfers. There model calculations which predict small contributions like ref. [20]. It will be a challenge to experimentalists to measure significantly such values.

The analysis of deuterium data taken by the $G^{0}$ and A4 collaborations will reduce the uncertainty in $\tilde{G}_{A}^{p}$ and hence also in in $G_{E}^{s}$ and $G_{M}^{s}$. In 2009 a new experimental program at the higher momentum transfer $Q^{2}=0.6 \mathrm{GeV}^{2}$ will performed both by the Happex and the A4 collaboration.

\section{References}

[1] B. Kaplan and A. Manohar, Nucl. Phys. B310, 527 (1988).

[2] X. Ji, Phys.Rev.Lett. 74, 1071-1074 (1995).

[3] E. Leader, Eur. Phys. J. A 32, 435-437 (2007).

[4] D. H. Beck and B. R. Holstein, Int. J. Mod. Phys. E 10, 1-41 (2001).

[5] M. J. Musolf et al., Phys. Rep. 239, 1 (1994).

[6] C. Amsler et al., Phys. Lett. B 667, 1 (2008).

[7] D. T. Spayde et al., Phys. Lett. B583, 79 (2004).

[8] F. E. Maas et al., Phys. Rev. Lett. 93, 022002 (2004).

[9] D. Armstrong et al., Phys. Rev. Lett. 95, 092001 (2005).

[10] A. Acha et al., Phys. Rev. Lett. 98, 032301 (2007).

[11] D. O. Riska, Eur. Phys. J. A32, 389 (2007).

[12] A. Silva et. al, Phys. Rev. D74, 054011 (2006).

[13] K. Goeke et al., Eur. Phys. J.A32, 393 (2007).

[14] D. B. Leinweber et al., Phys. Rev. Lett. 97, 022001 (2006)

[15] F. E. Maas et al., Phys. Rev. Lett. 94, 152001 (2005).

[16] F. E. Maas et al., Eur. Phys. J. A17, 339 (2003).

[17] J. Diefenbach et al. Eur. Phys. J. A32, 555-559 (2007).

[18] C. Weinrich et al. Eur. Phys. J. A24S2 129-130 (2005).

[19] I. Altarev et al., Nucl. Instr. Meth. A564, 13 (2006).

[20] P. Wang et al., arXiv:0807.0944 [hep-ph] (2008). 